\title{
Analysis of RFID Based Positioning Technique Using Received Signal Strength and Directional Antenna
}

\author{
Prajakta Shirke1, Amol Potgantwar $^{1}$, V. M. Wadhai ${ }^{2}$ \\ ${ }^{1}$ Department of Computer Engineering, Sandip Foundation's Sandip Institute of Technology and Research \\ Centre, Nashik, India \\ ${ }^{2}$ Sinhgad College of Academy, Pune, India \\ Email: prajaktashirke7@gmail.com, amol.potgantwar@sitrc.org, pune.wadhai.vijay@gmail.com
}

Received 6 February 2016; accepted 21 May 2016; published 24 May 2016

Copyright (C) 2016 by authors and Scientific Research Publishing Inc.

This work is licensed under the Creative Commons Attribution International License (CC BY).

http://creativecommons.org/licenses/by/4.0/

(c) (i) Open Access

\begin{abstract}
Automatic robot navigation is being utilized in many industries for the purpose of high speed work delivery. Color follower, fix path follower robots are current solution to this activities but dynamic path configuration is not possible in these robots. Hence new system proposes effective and fully dynamic path follower robots using RFID and directional antenna. Radio Frequency Identification (RFID) system permits automatic identification of objects with RFID tags using radio waves which have been widely used in mobile robot navigation, localization and mapping both in indoor and outdoor environment. This article presents a navigation strategy for autonomous mobile robot using passive RFID system. Proposed robot system is provided with RFID tag functionality which will load tag number and direction instruction. At some turning point, user will put RF tag, this tag will be read by $R F$ reader which is placed on robot. As per direction instruction robot will change the direction and reach to the destination. Also as per the movement, robot will send its GPS location to PC (Personal Computer) which will be displayed on PC. Hence main goal is to provide more reliable and low energy consumption based indoor positioning system which will be achieved using directional antenna.
\end{abstract}

\section{Keywords}

Global Positioning System (GPS), Indoor Positioning System (IPS), Radio Frequency Identification (RFID), Received Signal Strength (RSS)

\section{Introduction}

This article describes a navigation system for mobile robots which are assumed to move autonomously to a giv- 
en goal in man-made environments such as hallways in a building. Navigation services which usually depend on GNSS [1]-[3] are limited to be utilized in open areas with satellite signals. If users or robots are about to move in buildings, another approach must be used to navigate accurately. In this paper, Radio Frequency Identification (RFID) [4] is used to determine the location indoors. In RFID positioning, there are some common approaches to estimate the location. One method is based on signal power. Received signal strength (RSS) [4] [5] will be considered which presents the power of received signal as the measurement. The position will be calculated with certain methods based on the measurements. Several methods have been studied, such as cell based positioning, RFID location fingerprinting and the way using ranges to the tags calculated with RSSI [6]. Another particular method to approximate the location is based on the landmarks [7] [8]. In the landmark-based navigation, landmarks are needed to be set in the building, usually on certain doors and corners. A topological map with nodes corresponding to landmarks issued to do the navigation. System proposes a method using passive Radio Frequency Identification (RFID) tags as a landmark. The RFID tags are an unassertive i.e. passive type, non-contact, read-only memory system and can store a unique number for identification of the place. The tag data can be read from one meter away via electromagnetic waves. The tags are pasted on obstacles at any specific area. Robots just pass by the tags. The tags allow the acquisition of location information at mention speeds without the accurate control of robot positions for sensing the tags [9]. The passive RFID tags do not require an on-board power supply like a battery and generate operating power from the received electromagnetic waves. The tags are very thin and light. They therefore can be embedded easily in the environment and offer a virtually unlimited operational lifetime.

Indoor positioning technology has a wide range of applications at present; it can be used for positioning the object and personal. There are some effective methods including RFID (Radio Frequency Identification), Wi-Fi positioning [10]-[12]. RFID technology uses RF non-contact form of two-way communication, access to relevant data, automatic identification tag. It has the advantages such as high precision, strong anti-interference, non-line of sight, high security. Also it can identify the high-speed movement of label and can identify multiple tags, is becoming the preferred methods for indoor positioning system.

\section{Previous Measuring Techniques and Positioning Systems}

\subsection{Measuring Principles and Techniques}

Considering environmental factors such as reflecting surface, line-of-sight, continuous moving objects, size of the floor it is not easy to perform radio propagation in the indoor environment. For the multipath propagation of the low radio signals there is no fixed model defined yet. Hence for positioning objects in indoor environment it is better to use more than one type of positioning techniques for the better performance and result. The target location is estimated using geo positioning properties using triangulation. Triangulation generally has two derivations such as angulation and lateration. The lateration is also called as the range measurement technique which locates the objects by using differences from multiple reference points. The triangulation method performed with the help of the received signal strength. The positioning can be done by using the angle measurement techniques such as time of arrival (TOA), time difference of arrival (TDOA) and round trip time of flight (RTOF) [13]-[15].

\subsubsection{Lateration Techniques}

\section{1) TOA}

The TOA measurements can be done generally by using the three reference points with respect to the signals for $2 \mathrm{D}$ positioning. For the TOA based techniques one way prorogation time is measured by calculating the distance between signal transmitter and signal measuring device or unit. Different signaling techniques are used to measure the TOA such as ultra-wide band (UWB) or direct sequence spread spectrum (DSSS). The list square algorithm can be used to locate the target using nonlinear cost function. The TOA based techniques are active bat and cricket [16].

$$
R=\text { time } \times \text { speed }
$$

where speed is the traveling velocity of the signal. Time shows the quantity of time spent by the signal travelling from the transmitting to the in receipt of node and $R$ denotes the reserve connecting the transmit node and the being paid node. Since speed can be regard as a recognized invariable, $R$ can be compute by observe time. 


\section{2) TDOA}

TDOA considers the different signal travelling time from the particular signal transmitter towards the multiple receivers. Same as the TOA this method considers that the position of the base nodes is known. The drawback of synchronization of TOA is addressed by TDOA by synchronizing the base node clock and the target node clock. The TDOA method can be used in the emergency call on the highway and also in sound source localization by artificially intelligent robot.

$$
\frac{R}{C_{1}}-\frac{R}{C_{2}}=t 1-t 2
$$

In Equation (2), $C_{1}$ denotes the speed of single kind of signal, $C_{2}$ the speed of one more than one kind of signal, $t 1$ and $t 2$ the occasion for these two signal travelling from one node to the additional respectively, and $\mathrm{R}$ is the space between the signal transmitter and receiving unit.

\section{3) RTOF}

Round trip time of flight measures the signal travelling from signal transmitter to the signal measuring device or unit. The range mechanism of the RTOF is same as the TOA and the complete roundtrip propagation time is measured by the measuring device or unit. When properly synchronized, this RTOF technique can be for passive RFID technology where the readers can energize the passive RFID tags and measures the RTOF. The distance will be calculated as follows:

$$
R=\frac{\left(T_{R T}-\Delta t\right) \times \text { Speed }}{2}
$$

where $T_{R T}$ denotes the amount of time needed for a signal to go from one node to the other and back again, $\Delta t$ the predetermined time delay required by the hardware tool to work at the receiving node, and speed is the transmitting unit's speed.

\subsubsection{Angulation Technique (AOA Estimation)}

The angle between the transmitter and receiver line as well as the reference direction angle is measured by the angle of arrival technique (AOA). The accuracy of AOA depends on the rotating device and its angle. Also the accuracy also depends on the number of receivers in the receiving array. AOA is also known as the direction finding (DF) which can be measured by the antenna and the direction of the antenna.

\subsection{Positioning Systems}

\subsubsection{Global Positioning System (GPS)}

Object locations can be identify within a range of 1 to 5 meters [16] [17] by using most popular system known as the GPS [17] [18]. GPS is outdoor positioning method which uses triangulation approach for physically detecting locations with the help of satellites. Some GPS provides indoor positioning using highly attenuated signals but their positioning accuracy level is very low.

\subsubsection{Infrared Positioning System}

Active badges are the first indoor positioning system developed by AT \& T Cambridge which used diffuse IR technology [19]-[22] based system. This system based on network sensors working along with the IR code every 15 seconds and then uses the information based on triangulation theory. However, the shortcoming of IR technology such as short-range transmission and line-of-sight (LOS) [23] [24] does not allow the IR technology to be used in huge and complex regions.

\subsubsection{Ultrasonic Positioning System}

Active bats are the ultrasonic tracking [25]-[27] technique which is developed by AT \& T Cambridge which provides more accurate results than the previously defined IR positioning technique active badges. The ultrasonic tags are known as bats which are used to identify the objects. The ultrasonic bats emit periodic signals to the receivers which produces basic data for localization. This technique requires large amount of receivers with their sensitive alignments. Another ultrasonic positioning technique is cricket which developed in MIT laboratory as a location support system which uses radio frequency (RF) and the ultra-sonic technology based on 
Time-of-Flight (TOF) [28]. Real time tracking can be done with the rate of $1 \mathrm{~Hz}$. The drawback of the Cricket Location Support system was high cost that made it unaffordable to maximum users. Dolphin [29] [30] is another distributed object locating system which consist of distributed wireless sensor nodes. These nodes attached with the various indoor objects. Dolphin used to send and receive the ultrasonic signals [29]-[31] and RF which enables the positioning of objects.

\subsubsection{Received Signal Strength (RSS) Positioning System}

RSS is a measurement of the energy available in a received radio signal. In Existing Radio Based Measurement technique RSS [32] has been used widely in Indoor Positioning System. RSS determines the nature and characteristics of location fingerprints. The circuit voltage is measured between transmitters and receiving device which varies due to path interfaces and it is measured by received signal strength indicator (RSSI) [33] [34]. The distance measure is estimated by receiving node from the source node by determining the energy of received signal strength. It uses the path-loss factor to convert RSS into the distance measure and works on the principle that the signal power decreases if distance between nodes increases. According to the IEEE 802.11 standard system RSS is the corresponding received signal strength in a wireless environment. WaveLAN, RFID and Ultra wide band are the categories of the RF based signals [35]-[37].

1) WaveLAN Wireless Networking Technology

Positioning and tracking system in the building developed by using WaveLAN wireless networking technology [37] [38]. WaveLAN network interface card (NIC) is used for measuring signal ratio and signal strength. This system requires few number of base stations. The system must support WaveLAN NIC card for tracking object in a building this application not suitable for multi floored buildings [38].

\section{2) Ultra Wide Band (UWB) Technology}

Ultra wide band (UWB) [22] widely used for indoor location and tracking. The UWB system based on the Time of arrival (TOA) method which depends on the received signals. The signals calculated are transferred to the server computer where the transmitter is situated. UWB supports the multipath propagation to identify the active tag with accurate positioning.

\section{3) RFID Technology}

RFID initiates a one way wireless communication by use of noncontact and automatic identification technique that uses radio signals, puts the RFID tags on persons and objects. Wide variety of applications makes use of RFID such as automobile assemble industry, robotics industry, warehouse management area, even including persons. LANDMARC [39] AND SPOT ON are some well-known RFID location finding systems. This systems consist of RFID tags, RFID readers and the signal communication between the tags and readers. The basis of this system is the radio signal strength information from respective tags to reader and then uses the classifier algorithm to identify the user's position. Another system for tracking objects in indoor is RADAR [40]. This system uses signal propagation modelling for estimating the location using accuracy. Triangulation approach is used to identify the signal strength at the multiple receivers to identify the user's coordinates.

\section{Proposed Positioning System Based on Passive RFID and Directional Antenna}

For the purpose of avoiding the drawbacks of existing system this paper propose the new system with extended ideas. Proposed system will implement a navigation system for robot application. Here system will use RFID tags for the purpose of giving instructions to the robot. System will load some tag numbers and direction instructions in programmer. When robot system will be started to move, it will detect the path for navigation and start navigation on given path. At some point in given specified area, user has to put RF tag. This tag will be read by RF reader which is placed on robot. Every tag has one unique no. System will scan the number and its instruction for direction. As per direction instruction, robot will change the direction and reach to destination. Here system requires multiple tags to create random path. Also as per the movement, robot will send its GPS location to machine or personal computer (PC) and it will display position of the RFID tag on it. Here system will give forward, backward, left and right and stop instructions to robot.

\subsection{RFID Grid}

The RFID grid is an area that measures $120 \mathrm{~cm}$ by $120 \mathrm{~cm}$ in which the robot will be navigated. The RFID tags are place in a $5 \times 5$ configuration. The spacing between tags is $30 \mathrm{~cm}$. Horizontal rows and vertical columns are 
numbered 1 through 5. Figure 1 shows the layout of the RFID grid. This was deemed to be the optimal configuration based on the range of the RFID reader. During initial testing, the reader demonstrated a reading range of approximately $3 \mathrm{~cm}$. This limited the distance system could space the tags from each other. If the tags were spaced any further from each other, the probability of the robot not hitting a tag would increase.

\subsection{Navigation Algorithm}

Navigating in the grid is a systematic process. It is assumed that the robot is first placed on the outer edges of the RFID grid, facing the centre. The robot will then proceed to navigate itself and find the tag located in row 1 , column 3. The navigation algorithm starts by first determining what direction the robot is facing, and then selects the appropriate sub-algorithm.

\subsection{Direction Finding}

The flowchart to determining direction is shown in Figure 2. When the robot is first placed anywhere on the edge of the grid, facing the centre, the RFID microcontroller (The microcontroller tasked to direct the RFID reader to obtain tag information) obtains tag information from reader. This tag information is the identification number found on the tag. The RFID microcontroller then matches the retrieved tag information to a pre-assigned

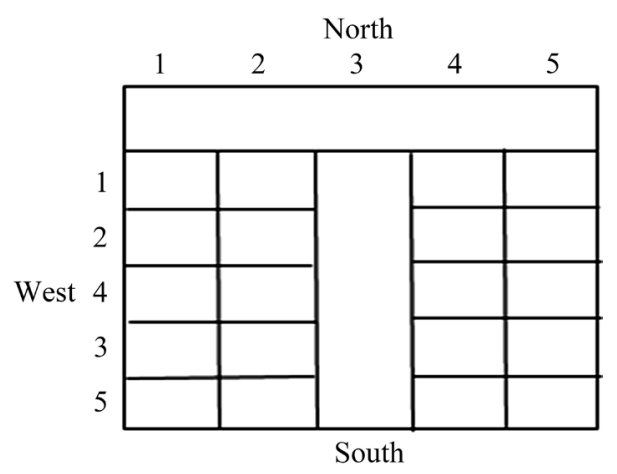

Figure 1. RFID grid.

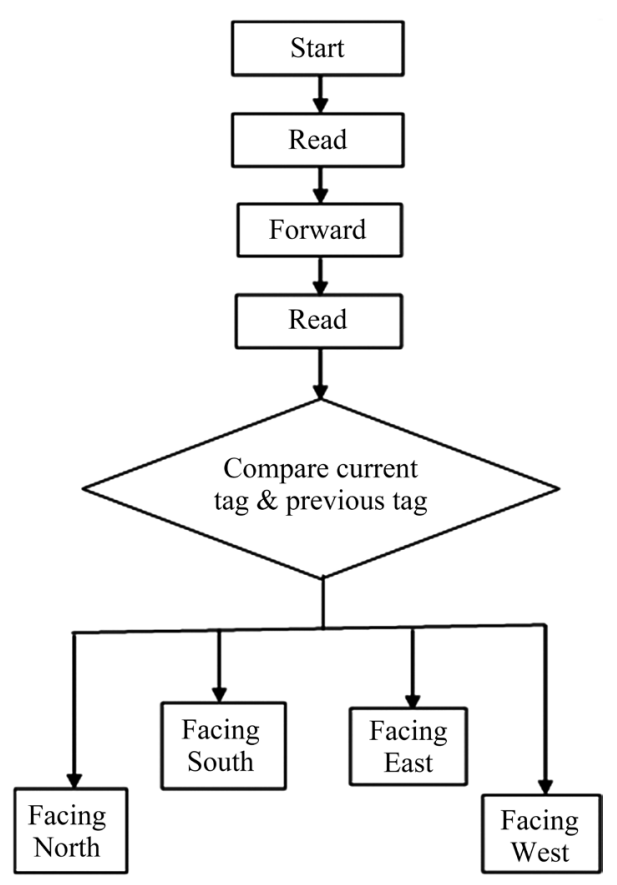

Figure 2. Direction finding flowchart. 
list of where this particular tag will be found on the grid. The tag value from the pre assigned list is the information that will be sent to the navigation microcontroller (The microcontroller tasked to perform the navigation algorithm). The converted tag information will now be labelled as the previous tag information and put into the navigation algorithm memory and it will be used later in the process. After the robot has retrieved and converted the initial tag information, it will proceed forward until it finds a new tag. The navigation microcontroller sends the command to the robot microcontroller (the microcontroller tasked to control the robot) which tells the robot to move forward. When a new tag is found, the RFID microcontroller tells the navigation microcontroller to inform to the robot microcontroller to stop. The RFID microcontroller will then retrieves and convert the new tag information and send it to the navigation microcontroller. This microcontroller will then label this new tag information as current tag information. After this process of obtaining previous and current tag information, the navigation algorithm can now determine what direction the robot has been placed.

\subsection{RFID (Radio Frequency Identification)}

RFID system has three basic components, RFID tags (transponders), RFID reader (interrogators), and Firmware. The firmware plant is a communication between the reader and tag to retrieve the tag serial (ID) number. The firmware also manages the communication with main controller to send the tag ID so that the controller can perform its required task using that information. The RFID reader can detect a RFID tag in its wireless range. Based on the operating principles, a tag can be characterized as active, semi-passive, and passive. A passive RFID tag is cheapest and least complex among the all three types. Passive tag does not have its own power source. It uses the Electromagnetic (EM) field from the reader to influence up its internal circuit.

\subsubsection{RFID Tag}

RFID tags can be also classified into three types: passive, active and semi-passive (also known as battery-assisted passive, or BAP). A typical RFID tag includes a microchip attached to a radio antenna mounted on a substrate. Although both active and passive tags use RF energy to interact with a reader, they are fundamentally different in the method of powering the tags. An active tag utilizes an internal power source, usually batteries, within the tag to continuously power the RF communication circuits and there tag, whereas a passive tag completely relies on RF energy transferred from the reader. Semi passive tags removes two key disadvantages of pure passive RFID tags, one is the lack of a continuous source of power for the circuits and another is the short reading range. Semi-passive tags are ideal for rapid development of customised RFID tags. There are two basic types of chips available for RFID tags: read-only and read-write. Read-only tags are cheaper and their needed infrastructure is also less expensive, they still deliver on one of the main promises of RFID, which is the minimization of operator involvement. The size of RFID tags varies largely with the purpose of applications.

\subsubsection{RFID Reader}

An RFID reader reads data from RFID tags and it acts as a conduit or bridge between the controller, middleware and RFID tags. The most important feature of a reader is its reading range. Reading range can be affected by a number of factors such as the frequency, the antenna gain, and orientation, polarisation of the reader antenna, the transponder antenna and the placement.

\subsection{Directional Antenna}

Directional antennas as the name implies refers to signal coverage in a specified direction. Unlike omnidirectional antennas, directional antenna must be aimed in the direction of the signal transmitter or receiver which can be for example a router or Wi-Fi hotspot. When it comes to aiming the antenna the user must be right on the signal for the best strength and quality. The way to ensure required aim is best to know the width of the reception cone in which the antenna has. A good way to start without seeing actual lobe data or knowing the specs is to judge by the $\mathrm{dBi}$ (decibel isotropic) rating. The higher the $\mathrm{dBi}$ rating, the narrower the reception cone. Knowing these data points one can then begin to narrow down the right antenna for system.

Figure 3 shows typical radiation pattern of directional antenna having gain of $21 \mathrm{dBi}$, a front-to-back ratio of $20 \mathrm{~dB}$ or a front-to-side ratio of $15 \mathrm{~dB}$. This means the gain in the backward direction is $1 \mathrm{dBi}$, and gain off the side is $6 \mathrm{dBi}$. In order to optimize the overall performance of a wireless LAN, it is important to understand how to maximize radio coverage with the appropriate antenna selection and placement. 


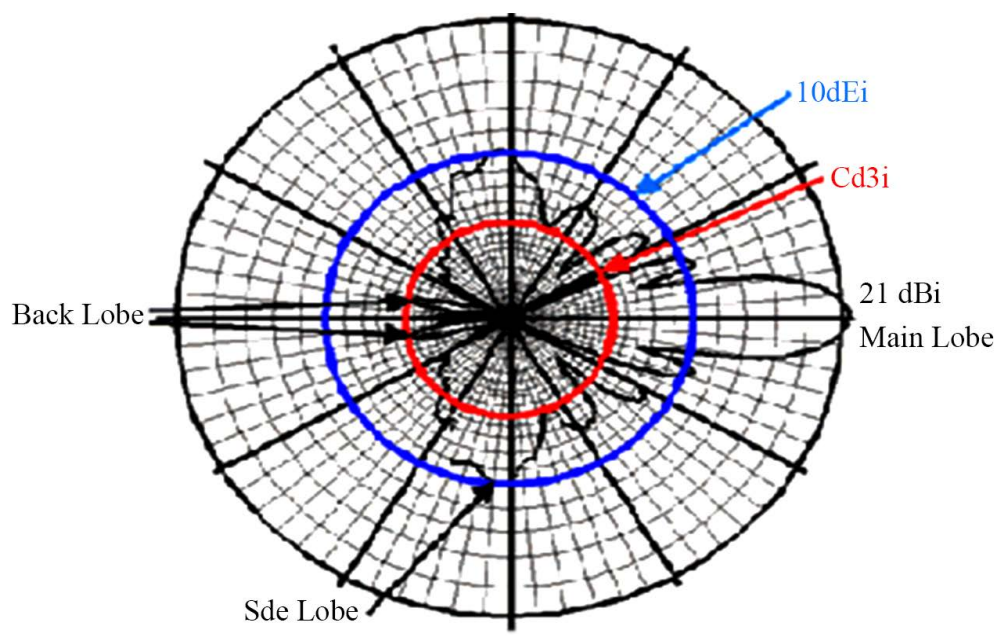

Figure 3. Typical radiation pattern of directional antenna.

\subsection{Indoor Effects}

Wireless propagation can be effected by reflection, refraction or diffraction in a particular environment. Diffraction is the bending of waves around the corners. RF waves can take multipath between the transmitter and receiver. A multipath is a combination of a primary signal and reflected, refracted or diffracted signal. So on the receiver side, the reflected signals combined with the direct signal can corrupt the signal or increase the amplitude of the signal, which depends on the phases of these signals. Because the distance travelled by the direct signal is shorter than the bounced signal, the time differential causes two signals to be received. These signals are overlapped and combined into a single one. In real life, the delay time i.e. time between the first received signal and the last echoed is the parameter used to signify multipath. The delay of the reflected signals is measured in nano seconds. The amount of delay time depends upon the amount of obstacles or infrastructure present between the transmitter and receiver. Therefore, delay in timing has more value for the manufacturing floor due to lot of metallic structure present as compared to the home environment. Overall, multipath limits the data rate or lowers the performance.

\section{Experimental Setup}

Figure 4 shows block diagram of the propose system which will estimate the position of mobile robot through the matching of the RFID tags. The proposed system utilizes RFID based localization using GPS receiver. The RFID tags are marked in floor and the robot has the RFID reader connected to it. Whenever the RFID reader passes near from a tag, it scans the data from tag. The position coordinate of the tag is previously stored in the PC. As per the data received from tag, position coordinate is approximated. The robot navigates in a dynamic path. ARM7 processor reads the RFID tag data and passes to the PC through serial port. In PC JAVA application matches the RFID data with predefined route map. According to the landmark and position coordinate the robot migrates through the dynamic path. Proposed system will be implemented using java jdk1.8 and Net Beans 8.0 on Pentium 4 (p4) Personal Computer (PC) having 1 GB of ram and 500 GB Hard Disk Drive (HDD) with Windows Operating System. The hardware system will be consist of selected ARM 7 processor, RFID reader and tags along with directional antenna. Block Diagram of the system will be as follows.

\section{Expected Result}

Proposed system will analyse various parameters such as accuracy, precision, scalability, and energy consumption. Environmental effects on signal and angle loss. System expects the fully dynamic navigation in error free environment. All the results will be compared with existing solutions such as fix path follower and colour follower. Angle loss in directional antenna (DA) will be reduced by adjusting angle of antenna and displacement factor. Use of RFID and DA will become low cost solution for current problem. This system can be used across many real time applications such as automobile industries, transport systems, packaging and logistics industries. 


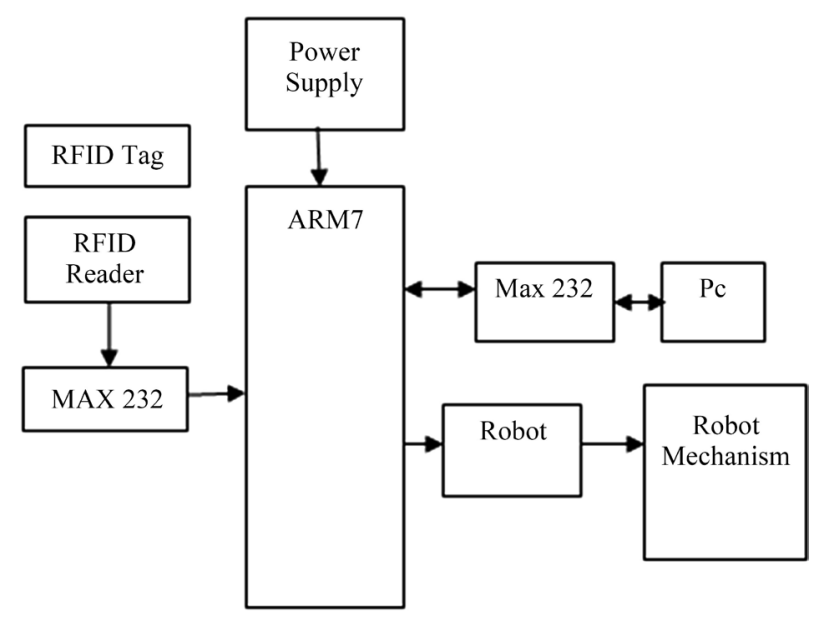

Figure 4. Block diagram of propose system.

\section{Conclusion}

Based on the analysis, this method shows effective results in providing good migration system for mobile robot in indoor environment. The system provides the data for both position and orientation of the robot. No issue what the initial orientation of the robot is, it can orient itself successfully to face to the goal location. To enhance the accuracy of active RFID indoor location system, an adaptive self-correction location algorithm based on RSS improvement is presented. The main parts of the system are RFID readers, tags, directional antenna and the sensors, which enable the robot to locate itself accurately in given environment.

\section{References}

[1] Russian Institute of Space Device Engineering (2008) Global Navigation Satellite System GLONASS—Interface Control Document. Version 5.1, Moscow, Russia.

[2] Gu, Y., Lo, A. and Niemegeers, I. (2009) A Survey of Indoor Positioning Systems for Wireless Personal Networks. IEEE Communications Surveys \& Tutorials, 11, 13-32.

[3] Szunny, R. and Modelski, J. (2005) Neural Networks in Indoor Positioning System Based on Power Delay Profile. The International Conference on “Computer as a Tool”, EUROCON 2005, Belgrade, 21-24 November 2005, 1726-1729. http://dx.doi.org/10.1109/EURCON.2005.1630307

[4] Xiong, Z., Song, Z., Sclera, A., Ferrea, E., Sottile, F., Brizzi, P., Tomsai, R. and Spirito, M.A. (2013) Hybrid WSN and RFID Indoor Positioning and Tracking System. EURASIP Journal on Embedded Systems, 2013, 6.

[5] Liu, H., Darabai, H., Banerjee, P. and Liu, J. (2007) Survey of Wireless Indoor Positioning Technique and Systems. IEEE Transactions on Systems, Man, and Cybernetics, Part C (Applications and Reviews), 37, 1067-1080. http://dx.doi.org/10.1109/TSMCC.2007.905750

[6] Pei, C., Cai, Y. and Ma, Z. (2009) An Indoor Positioning Algorithm Based on Received Signal Strength of WLAN. Pacific-Asia Conference on Circuits, Communications and Systems, Chengdu, 16-17 May 2009, 516-519. http://dx.doi.org/10.1109/PACCS.2009.65

[7] Lee, B.-G., Lee, Y.-S. and Chung, W.-Y. (2008) 3D Navigation Real Time RSSI-Based Indoor Tracking Application. Journal of Ubiquitous Convergence Technology, 2, 67-77.

[8] Lionel, M.N.I., Liu, Y.H., Lau, I.C. and Patil, A.P. (2003) LANDMARC: Indoor Location Sensing Using Active RFID. Proceedings of the 1st IEEE International Conference on Pervasive Computing and Communications, Fort Worth, 26-26 March 2003, 407-415.

[9] Shi, H.Y. (2012) A New Weighted Centroid Localization Algorithm Based on RSSI. International Conference on Information and Automation, Shenyang, 6-8 June 2012, 137-141.

[10] Hightower, J., Want, R. and Borriello, G. (2000) SpotON: An Indoor 3D Location Sensing Technology Based on RF Signal Strength. UW CSE00-02-02.

[11] Potgantwar, A.D. and Wadhai, V.M. (2011) Location Based System for Mobile Devices with Integration of RFID and Wireless Technology-Issues and Proposed System. 2011 International Conference on Process Automation, Control and Computing, Coimbatore, 20-22 July 2011, 1-5. 
[12] Wang, C.L. and Chiou, Y.S. (2006) An Adaptive Positioning Scheme Based on Radio Propogation Modelling for Indoor WLANs. 63rd IEEE Vehicular Technology Conference, Melbourne, 7-10 May 2006, 2676-2680.

[13] Paul, A.S. and Wan, E.A. (2009) RSSI-Based Indoor Localization and Tracking Using Sigma-Point Kalman Smoothers. IEEE Journal of Selected Topics in Signal Processing, 3, 860-873. http://dx.doi.org/10.1109/JSTSP.2009.2032309

[14] Dong, Z., Wu, Y. and Sum, D. (2013) Data Fusion of the Real-Time Positioning System Based on RSSI and TOF. 5th International Conference on Intelligent Human-Machine Systems and Cybernetics, 2, 503-506. http://dx.doi.org/10.1109/ihmsc.2013.267

[15] Günther, A. and Hoene, C. (2005) Measuring Round Trip Times to Determine the Distance between WLAN Nodes. 4th International IFIP-TC6 Networking Conference, Waterloo, 2-6 May 2005, 768-779.

[16] Pascucci, F. and Ulivi, G. (2002) An Outdoor Navigation System Using GPS and Inertial Platform. IEEE/ASME Transactions on Mechatronics, 7, 134-142. http://dx.doi.org/10.1109/TMECH.2002.1011250

[17] Tsubouchi, T., Shigematsu, B., Maeyama, S. and Yuta, S. (2003) Outdoor Navigation of a Mobile Robot between Buildings Based on DGPS and Odometry Data Fusion. IEEE International Conference on Robotics and Automation, 2, 1050-4729.

[18] Department of Defence United States of America and GPS Navstar (2008) Global Positioning System Standard Positioning Service Performance Standards. 4th Edition, Department of Defence United States of America and GPS Navstar.

[19] Kennedy, S.H. and Martell, J. (2006) Architecture and System Performance of SPAN NovAtel's GPS/INS Solution. Proceedings of IEEE/ION PLANS 2006, San Diego, 23-25 April 2006.

[20] Want, R., Hopper, A., Falcao, V. and Gibbons, J. (1992) The Active Badge Location System. ACM Transactions on Information Systems, 40, 91-102. http://dx.doi.org/10.1145/128756.128759

[21] Want, R., Schilit, B., Adams, N., Gold, R., Gold-Berg, D., Petersen, K., Ellis, J. and Weiser, M. (1997) The Parctab Ubiquitous Computing Experiment. In: Imielinski, T., Ed., Book Chapter: Mobile Computing, Chapter 2, Kluwer Publishing, 45-101.

[22] Ward, A., Jones, A. and Hopper, A. (1997) A New Location Technique for the Active Office. IEEE Personal Communication Magazine, 4, 42-47.

[23] Geunho, L. and Young, C.N. (2011) Low-Cost Dual Rotating Infrared Sensor for Mobile Robot Swarm Applications. IEEE Transactions on Industrial Informatics, 7, 277-286. http://dx.doi.org/10.1109/TII.2011.2121078

[24] Krumm, J., Harris, S., Meyers, B., Brumitt, B., Hale, M. and Shafer, S. (2000) Multi Camera Multi-Person Tracking Easy Living. 3rd IEEE International Workshop on Visual Surveillance, Dublin, 1 July 2000, 3-10. http://dx.doi.org/10.1109/VS.2000.856852

[25] Priyantha, N.B. (2005) The Cricket Indoor Location System. Ph.D. Thesis, Massachusetts Institute of Technology, Cambridge, $199 \mathrm{p}$.

[26] Fukuju, Y., Minami, M., Morikawa, H. and Aoyama, T. (2003) DOLPHIN: An Autonomous Indoor Positioning System in Ubiquitous Computing Environment. IEEE Workshop on Software Technologies for Future Embedded Systems, Hakodate, 15-16 May 2003, 53-56.

[27] Li, T.H.S., Yeh, Y.C., Wu, J.D., Hsiao, M.Y. and Chen, C.Y. (2010) Multifunctional Intelligent Autonomous Parking Controllers for Carlike Mobile Robots. IEEE Transactions on Industrial Electronics, 57, 1687-1700. http://dx.doi.org/10.1109/TIE.2009.2033093

[28] Kyoungmin, L. and Kyun, C.W. (2009) Effective Maximum Likelihood Grid Map with Conflict Evaluation Filter Using Sonar Sensors. IEEE Transactions on Robotics, 25, 887-901. http://dx.doi.org/10.1109/TRO.2009.2024783

[29] Orr, R.J. and Abowd, G.D. (2000) The Smart Floor: A Mechanism for Natural User Identification and Tracking. GVU Technical Report GIT-GVU-00-02 (Full Paper).

[30] Minami, M., Fukuju, Y., Hirasawa, K., Yokoyama, S., Mizumachi, M., Morikawa, H. and Aoyama, T. (2004) DOLPHIN: A Practical Approach for Implementing a Fully Distributed Indoor Ultrasonic Positioning System. In: Davies, N., Mynatt, E.D. and Siio, I., Eds., UbiComp 2004: Ubiquitous Computing, Springer, Berlin, 347-365.

[31] Hazas, M. and Hopper, A. (2006) A Novel Broadband Ultrasonic Location System for Improved Indoor Positioning. IEEE Transactions on Mobile Computing, 5, 536-547. http://dx.doi.org/10.1109/TMC.2006.57

[32] Lorincz, K. and Welsh, M. (2005) MoteTrack: A Robust, Decentralized Approach to RF-Based Location Tracking. Proceedings of the International Workshop on Location and Context-Awareness (LoCA 2005) at Pervasive 2005, May 2005.

[33] Lau, E.-E.-L. and Chung, W.-Y. (2007) Enhanced RSSI-Based Real-Time User Location Tracking System for Indoor and Outdoor Environments. International Conference on Convergence Information Technology, Gyeongju, 21-23 November 2007, 1213-1218. http://dx.doi.org/10.1109/iccit.2007.253 
[34] Collin, J., Mezentsev, O. and Lachapelle, G. (2003) Indoor Positioning System Using Accelerometry and High Accuracy Heading Sensors. Proceedings of GPS/GNSS 2003 Conference (Session C3), Portland, 9-12 September 2003.

[35] Harter, A., Hopper, A., Steggles, P., Ward, A. and Webster, P. (1999) The Anatomy of a Context Aware Application. Proceedings of the 5th Annual ACM/IEEE International Conference on Mobile Computing and Networking, August 1999, 59-68.

[36] Tesoriero, R., Tebar, R., Gallud, J.A., Lozano, M.D. and Penichet, V.M.R. (2010) Improving Location Awareness in Indoor Spaces Using RFID Technology. Expert Systems with Applications, 37, 894-898. http://dx.doi.org/10.1016/j.eswa.2009.05.062

[37] Harter, A. and Hopper, A. (1994) A Distributed Location System for the Active Office. IEEE, Network.

[38] Bahl, P. and Padmanabhan, V.N. (1999) User Location and Tracking in an In-Building Radio Network. Microsoft Research Technical Report: MSR-TR-99-12.

[39] Pugh, J., Raemy, X., Favre, C., Falconi, R. and Martinoli, A. (2009) A Fast Onboard Relative Positioning Module for Multirobot Systems. IEEE/ASME Transactions on Mechatronics, 14, 151-162. http://dx.doi.org/10.1109/TMECH.2008.2011810

[40] Bahl, P. and Padmanabhan, V.N. (2000) RADAR: An In-Building RF Based User Location and Tracking System.: Proceedings of IEEE INFOCOM 2000, Israel, March 2000. 